\title{
Sobre la identidad taxonómica de Brachistosternus peruvianus Piza, 1974 (Scorpiones: Bothriuridae)
}

\section{On the taxonomic identity of Brachistosternus peruvianus Piza, 1974 (Scorpiones: Bothriuridae)}

\author{
José A. Ochoa
}

Departamento de Zoologia Instituto de Biociências, Universidade de São Paulo, Rua do Matão, travessa 14, 101, São Paulo, SP, 05508-900, Brasil.Email: jaochoac2000@ yahoo.com

\begin{abstract}
Resumen
Se establece la verdadera identidad de Brachistosternus peruvianus Piza, 1974, un controversial escorpión andino, previamente confundido con Brachistosternus andinus Chamberlin, 1916. Ambas especies habitan en valles interandinos del sur del Perú, y pertenecen al subgénero Brachistosternus (Ministernus) Francke. Se presenta una detallada redescripción para ambas especies basada en la morfología externa, patrones de pigmentación y caracteres del hemispermatóforo. Se reportan también nuevos registros y una breve discusión sobre la ecología y distribución de estas dos especies.
\end{abstract}

Palabras clave: Scorpiones, Bothriuridae, Brachistosternus, Perú, Andes.

\section{Abstract}

The true identity of Brachistosternus peruvianus Piza, 1974, a controversial Andean scorpion previously confused with Brachistosternus andinus Chamberlin, 1916, is established. Both species inhabit inter Andean valleys of southern Peru and belong to the subgenus Brachistosternus (Ministernus) Francke. A detailed redescription of both species based on external morphology, pigmentation pattern and hemispermatophore features are provided. New records for these species, along with brief discussions of the ecology and distribution are also reported.

Keywords: Scorpiones, Bothriuridae, Brachistosternus, Peru, Andes.

\section{Introducción}

En 1974 fue descrita la especie de escorpión Brachistosternus peruvianus Piza, para el departamento de Apurímac en el sur del Perú (Piza 1974), desde entonces la posición taxonómica de esta especie fue largamente considerada como incierta y su validez fue cuestionada por varios autores (Francke 1977, Maury 1978, Ochoa \& Acosta 2002). La causa principal de esta confusión radica en que la descripción original no presenta detalles suficientes que permitan discriminarla de otras especies dentro del género Brachistosternus Pocock, 1893. Maury (1978) supone por algunos datos de la descripción, las fotos, y procedencia del material, que podía tratarse de un sinónimo posterior de Brachistosternus andinus Chamberlin, 1916, que habita los valles interandinos en el departamento de Cusco (Ochoa 2005); no obstante, dicho autor no tuvo acceso al material tipo de $B$. peruvianus, por tanto no formalizó la sinonimia. Esta suposición fue tomada como válida por Lowe \& Fet (2000) en su Catálogo de escorpiones, quienes erróneamente ubican a $B$. peruvianus como sinónimo de B. andinus, atribuyendo la sinonimia a Maury (1978).

Según la descripción original (Piza 1974), el material tipo (un macho y una hembra sintipos) fueron depositados en la Escuela Superior de Agricultura "Luiz de Queiroz" en Piracicaba del Estado de Sáo Paulo, Brasil, sin embargo, en el Catálogo de Lowe \& Fet (2000) mencionan erróneamente que dicho material estaría depositado en el Museo de Zoologia de la Universidade de São Paulo (MZUSP). Es muy probable que en la actualidad dicho material se encuentre perdido.

La revisión de la colección de escorpiones del Museo de Historia Natural de la Universidad del Cusco (MHNC), nos permitió detectar algunos ejemplares etiquetados como Brachistosternus peruvianus colectados en 1969 procedentes del departamento de Apurímac. De acuerdo con O. Ochoa M. (com. pers.), quien colectó y envió el material a Piza, se remitieron ejemplares de dos localidades del departamento de Apurímac: series MHNC 107-111 de la localidad de Pachachaca y series MHNC 101-104 de la localidad de Cunyac; por tal razón el material tipo correspondería a una de las dos localidades. Lamentablemente Piza (1974) no menciona localidad, fecha de colecta ni número de serie. En el MHNC se encuentran parte del lote correspondiente a la localidad de Pachachaca (series 112-114, 116-118, 123-125), lo cual permitió, además de material adicional procedente de otras localidades en el departamento de Apurímac, diferenciar $B$. peruvianus de $B$. andinus y confirmar su status como especie válida. Maury (1978) confunde ambas especies probablemente porque no tuvo acceso a ejemplares machos adultos de los verdaderos $B$. andinus, incluso el dibujo que ofrece del hemiespermatóforo (figs. 11, 12 de Maury 1978) sin duda corresponde a B. peruvianus.

En un trabajo anterior (Ochoa 2005) ya habíamos considerado ambas entidades como especies distintas, las cuales además presentan distribución alopátrica en el sur del Perú; ello fue ratificado posteriormente por Ojanguren Affilastro \& Ramírez (2009), quienes tuvieron acceso a la mayoría de los especímenes estudiados por Maury. En el presente estudio presentamos una descripción detallada de ambas especies de acuerdo a los estándares actuales.

Brachistosternus andinus y B. peruvianus, pertenecen al subgénero Brachistosternus (Ministernus) Francke, 1985 (Ojanguren Affilastro \& Ramírez 2009) el cual se caracteriza principalmente por tener 9 a 13 tricobotrias ventrales y 5 tricobotrias externas basales en la pinza del pedipalpo, patela con 3 tricobotrias ventrales y 12 externas (falta la $e s b_{2}$ ); quelíceros pequeños de cuerpo corto con un solo diente subdistal en el dedo móvil. El subgénero incluye dos especies más: $B$. ferrugineus (Thorell, 1876) y B. simoneae Lourenço, 2000; la primera es una especie que tiene distribución chaqueña en Bolivia, Argentina, Paraguay y SO de Brasil (Maury 1973, 1974, 1979; Acosta \& Maury 1998; Acosta \& Ochoa 2002; Ojanguren Affilastro \& Ramírez, 


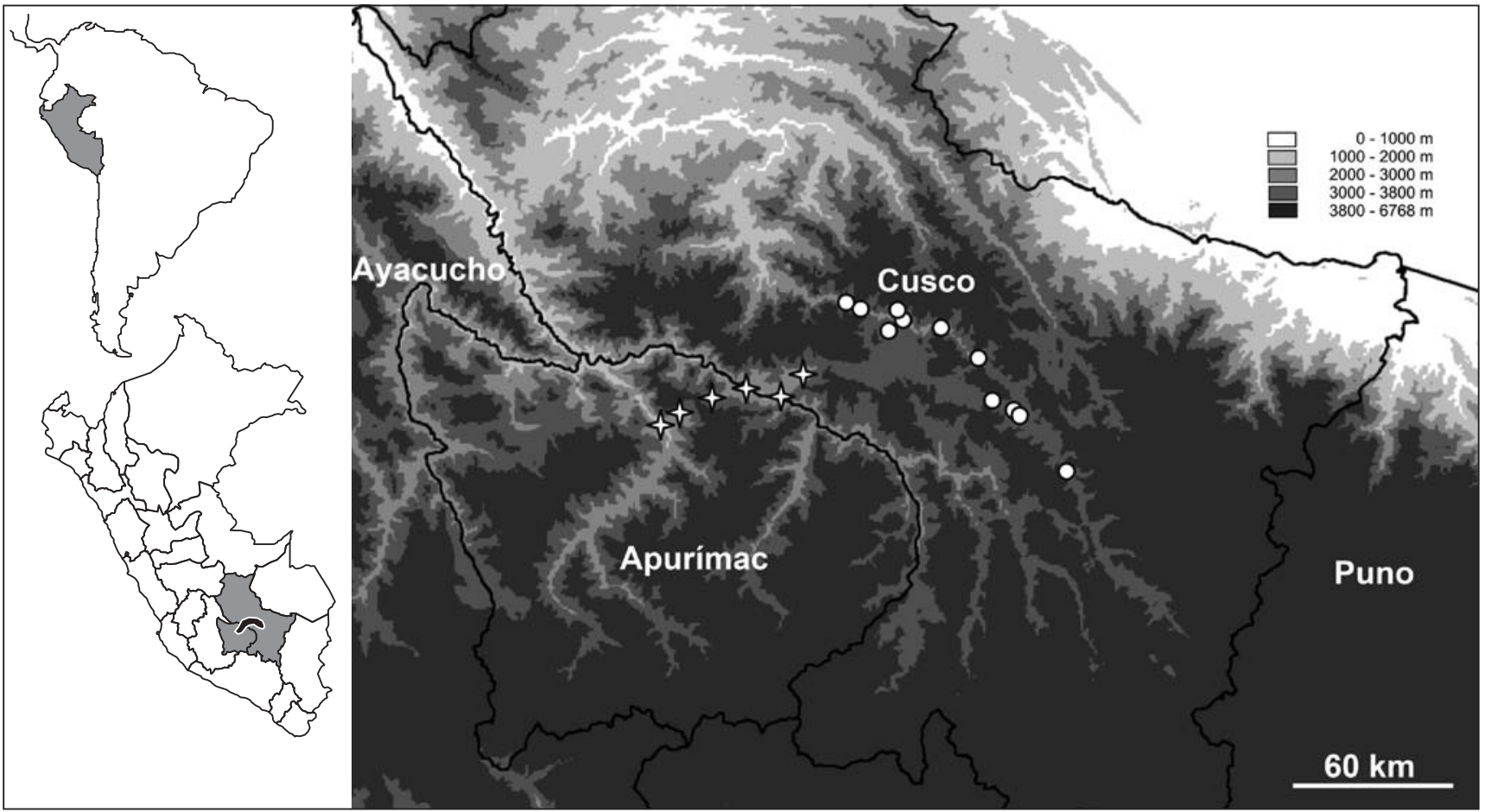

Figura 1. Mapa de distribución de Brachistosternus andinus Chamberlin, 1916 (círculos) y Brachistosternus peruvianus Piza, 1974 (estrellas), en los departamentos de Apurímac y Cusco en el sur de Perú.

2009); B. simoneae fue descrito para el estado de Goiás en Brasil en ambientes de Cerrado (Lourenço 2000), aunque se sospecha que se trata de un sinónimo de $B$. ferrugineus (A. Ojanguren Affilastro y C. Mattoni com. pers.); mientras que B. andinus y B. peruvianus, se encuentran presentes en valles interandinos del sur del Perú (Ochoa 2005).

\section{Materiales y métodos}

El material mencionado en el presente trabajo corresponde a las siguientes instituciones y museos: AMNH: American Museum of Natural History, New York, USA; CDA: Cátedra de Diversidad Animal I, Facultad de Ciencias Exactas, Físicas y Naturales, Universidad Nacional de Córdoba, Argentina. FMNH: Field Museum of Natural History, Chicago, USA. MHNC: Museo de Historia Natural, Facultad de Ciencias Biológicas, Universidad Nacional de San Antonio Abad del Cusco, Perú. ESALQ: Escola Superior de Agricultura "Luiz de Queiroz", Universidad de São Paolo, Piracicaba, Brasil.

Para la terminología de la morfología general utilizamos los trabajos de Vachon (1952) y Stahnke (1970); Prendini (2000) para las carenas del pedipalpo: interno mediana (IM), digital (D), dorsal interna (DI), dorsal mediana (DM), dorsal externa (DE), dorsal marginal (DMA), dorsal secundaria (DS), externa (E), externa mediana (EM), ventral externa (VE), ventral mediana (VM), ventral interna (VI), proceso dorsal de la patela (DPP), proceso ventral de la patela (VPP); Ochoa et al. (2010) para la terminología de las carenas del metasoma: dorsal submediana (DSM), dorsal lateral (DL), lateral supramediana (LSM), lateral mediana (LM), lateral inframediana (LIM), ventral lateral (VL), ventral submediana (VSM), ventral mediana (VM). Para la terminología de la tricobotriotaxia seguimos el trabajo de Vachon (1974), y Maury (1974) para el hemiespermatóforo.

Las ilustraciones fueron realizadas utilizando un estereomicroscopio Leica con cámara lúcida. Las medidas son presentadas en milimetros siguiendo la metodologia de Sissom et al. (1990). El mapa de distribución fue generado con el programa DIVAGIS Version 5.4 (http://www.diva-gis.org/) superponiendo los puntos y coordenadas de las localidades de colecta en una base de datos digital del CGIAR Consortium for Spatial Information (CGIAR-CSI) disponible en http://srtm.csi.cgiar.org.

\section{Taxonomía}

FAMILIA BOTHRIURIDAE SIMON, 1880

Brachistosternus POCOCK, 1893

Brachistosternus (MINISTERNUS) Francke, 1985

\section{Brachistosternus (Ministernus) andinus Chamberlin, 1916}

(figs. 2-11)

Brachistosternus andinus Chamberlin, 1916: 179; Mello-Leitão, 1931: 94, 1932: 34, 1945: 225; Aguilar \& Meneses, 1970: 2; Kovařík, 1998: 101; Ochoa \& Acosta, 2002: 2; Ochoa, 2005: 61, tabla 2; Rein, 2007: 3, 7; Ochoa \& Prendini, 2010: 43; Ojanguren Affilastro \& Ramírez, 2009: 188, 192, figs. 1, 2, 3A.

Brachistosternus (Microsternus) andinus: Maury, 1973 (part): 251, 1974 (part): 81, 1978 (part): 23, figs. 1-12, tabla I; Francke, 1977: 75; Del Castillo, 1986 (part): 90, 91.

Brachistosternus (Ministernus) andinus: Lowe \& Fet, 2000 (part): 52; Ochoa, 2003: 55; 2005: 54, 58, 62, fig. 4; Ojanguren Affilastro et al., 2007: 5, tabla 1; Ojanguren Affilastro \& Ramírez, 2009: 188, 192.

Serie típica. Holotipo hembra juvenil (MCZ 124), PERÚ, Departamento Cusco: Ollantaytambo [13ำ $15^{\prime} \mathrm{S} 72^{\circ} 15^{\prime} \mathrm{W}$, $2800 \mathrm{~m}$ ], 20 julio 1911, R. Foote.

Diagnosis. Especie perteneciente al subgénero Brachistosternus (Ministernus), dentro del cual la especie más cercana es $B$. peruvianus, con la que incluso fue confundida. Ambas entidades se diferencian principalmente por detalles en el hemiespermatóforo: la apófisis cilíndrica es más corta en $B$. andinus, en la que 

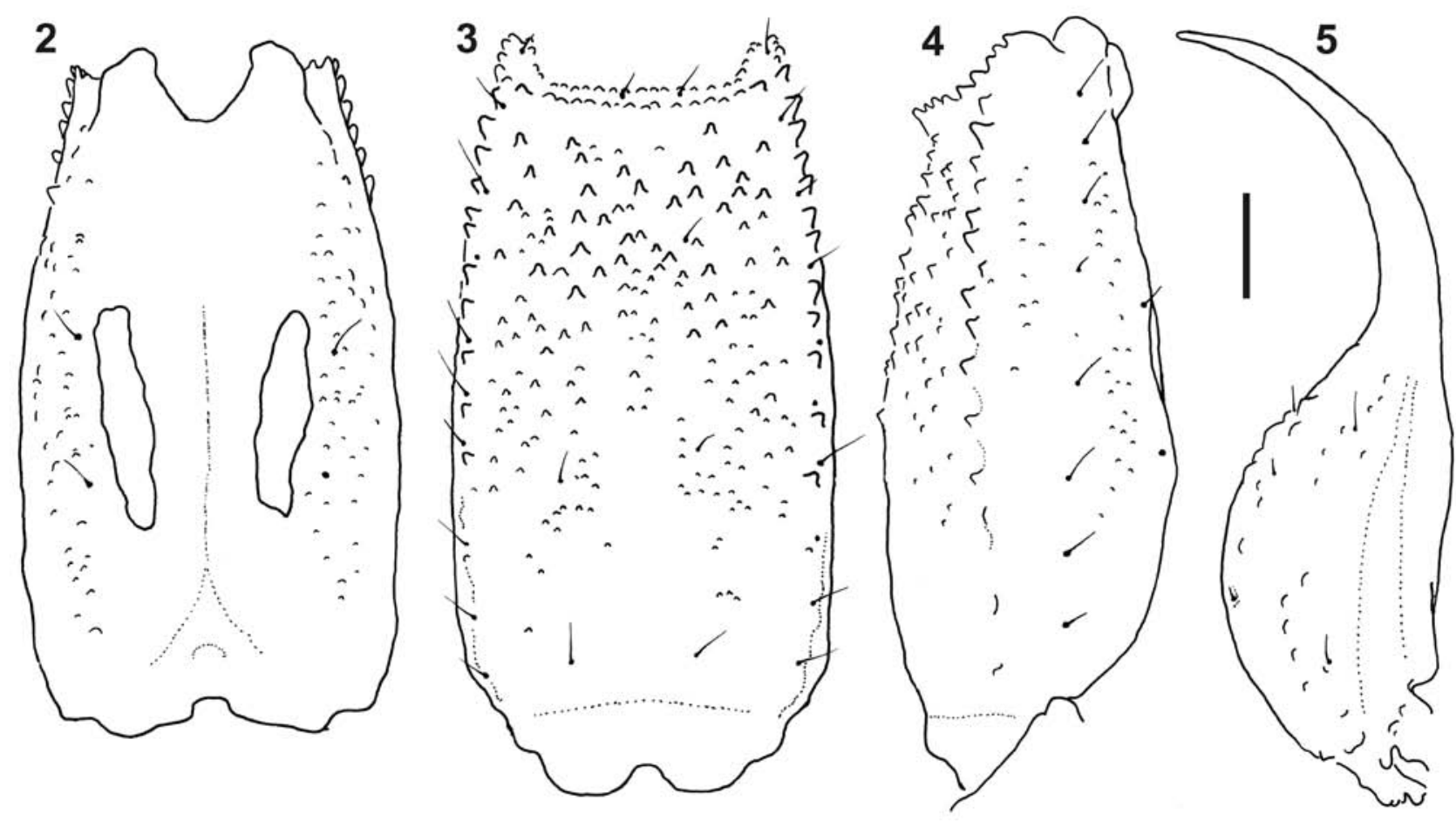

Figuras 2-5. Brachistosternus andinus Chamberlin, 1916, macho (MHNC). 2-4. Segmento caudal V, 2, vista dorsal; 3, vista ventral, 4, vista lateral. 5. Telson, vista lateral. Escala $1 \mathrm{~mm}$.

apenas sobrepasa el lóbulo interno (fig. 9); mientras que en $B$. peruvianus dicha apófisis es alargada y sobrepasa ampliamente el lóbulo interno (fig. 12); además la lámina distal en B. peruvianus es más alargada y presenta una curvatura en la parte media, la cual no es evidente en $B$. andinus. En cuanto a caracteres externos, la principal diferencia radica en el número de dientes pectíneos: en $B$. andinus varía entre 24-28 para las hembras y 27-30 para machos, mientras que en $B$. peruvianus, varía entre 34 y 36 en hembras y entre 35 y 40 en machos; adicionalmente, el número de tricobotrias ventrales de la pinza del pedipalpo varía en $B$. andinus de 9 a 11 y en $B$. peruvianus de 11 a 13 . Como datos complementarios los índices largo/alto del telson (media de 3,68 en $\widehat{\partial}$ y 3,25 en $\bigcirc$ para $B$. andinus; 3,94 en $\widehat{\partial}$ y 3,65 en $q$ para B. peruvianus); así como los de largo/ancho (media de 3,23 en ô y 3,76 en $q$ para $B$. andinus; 3,32 en $\delta$ y 3,93 en 9 para B. peruvianus) y largo/alto de la pinza del pedipalpo (media de 2,74 en ổ y 3,12 en $ᄋ$ para B. andinus; 2,90 en $\sigma^{\lambda}$ y 3,27 en 우 para $B$. peruvianus) presentan valores ligeramente mayores en $B$. peruvianus. Por último, en cuanto al tamaño corporal, los ejemplares de $B$. andinus son de menor talla, los adultos miden entre 39 a $52 \mathrm{~mm}$, mientras que en $B$. peruvianus entre 61 a $78 \mathrm{~mm}$. Comparaciones con $B$. ferrugineus y B. simoneae ver diagnosis correspondiente a $B$. peruvianus.

\section{Redescripción}

Coloración y patrón de pigmentación: Color general amarillo castaño, con manchas cafés oscuras; patas y parte ventral del prosoma y mesosoma amarillentas, peines blanquecinos. Caparax en la mayoría de los ejemplares con pigmento reticular hacia los laterales y ángulos latero-posteriores, dejando en la parte mediana una amplia zona sin pigmento desde el borde anterior al posterior, excepto la cúpula ocular que es negruzca; no obstante pocos ejemplares pueden presentar algunas manchas de pigmen- to hacia el borde anterior y una pigmentación tenue en toda la zona central adyacente a la cúpula ocular, pero el pigmento lateral siempre es más evidente. Tergitos I-VI con dos manchas laterales de pigmento, área mediana sin manchas o con pigmento tenue; en algunos ejemplares el borde posterior de cada tergito suele presentar pigmento. Pretergitos pigmentados. Tergito VII sin pigmento, o con ligeras manchas laterales. Segmentos caudales I-IV sin pigmentación evidente, salvo tenues manchas ventrales en los segmentos III-IV que aparentan tres bandas longitudinales que confluyen distalmente. Segmento caudal V: cara dorsal y laterales con algunas manchas reticulares no muy marcadas; cara ventral con abundante pigmento, con tres bandas que confluyen en la mitad distal del segmento, acompañado de retículo paramediano que también confluye. Telson con ligeras manchas ventrales, aguijón oscurecido. Quelíceros con pigmento cerca de la base de los dedos, los cuales están pigmentados. Pedipalpos y patas con algunas manchas no muy evidentes y en la mayoría de los ejemplares la pinza es despigmentada.

Morfología. Longitud total: machos 39,0-48,5 mm; hembras hasta 52,8 (medidas de macho y hembra en Tabla 1).

Quelíceros: Con un diente subdistal en el dedo móvil. Tegumento liso con algunas setas ventrales en la base del dedo fijo.

Caparax: Borde anterior del caparax con una ligera prominencia mediana; surco longitudinal anterior poco marcado, surcos longitudinal posterior y laterales posteriores más evidentes. Cúpula ocular ligeramente desarrollada, ubicada aproximadamente en la parte central del caparax. Tegumento finamente granuloso en los machos, con gránulos ligeramente más notorios en los ángulos latero-posteriores, (hembras menos granulosas).

Pedipalpos: Pedipalpos (figs. 6-8): Fémur con las carenas DI, DE, IM, VI presentes pero obsoletas y con gránulos menores; 

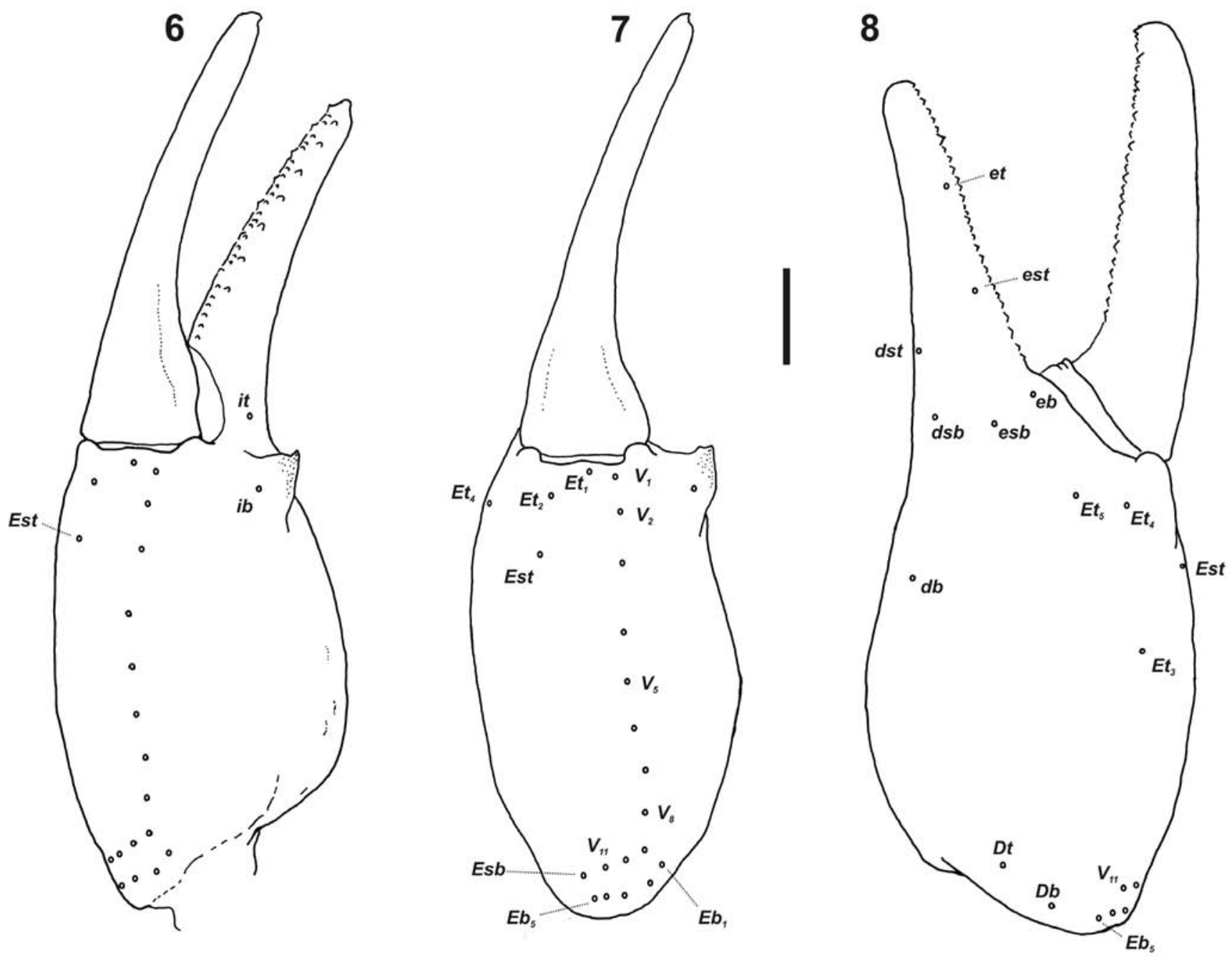

Figuras 6-8. Brachistosternus andinus Chamberlin, 1916, macho (MHNC). Pinza del pedipalpo derecho. 6, vista ventromedial; 7, vista ventral, 8, vista externa. Escala $1 \mathrm{~mm}$.

Tabla 1. Medidas en mm de un macho y una hembra (MHNC) de Brachistosternus andinus Chamberlin, 1916 y Brachistosternus peruvianus Piza, 1974.

\begin{tabular}{|c|c|c|c|c|}
\hline & \multicolumn{2}{|c|}{ Brachistosternus andinus } & \multicolumn{2}{|c|}{ Brachistosternus peruvianus } \\
\hline & Macho & Hembra & Macho & Hembra \\
\hline Longitud total & 41,6 & 46,7 & 69,7 & 64,1 \\
\hline Caparax, largo & 5,4 & 6,5 & 8,5 & 8,8 \\
\hline ancho anterior / posterior & $3,9 / 5,6$ & $4,6 / 7,2$ & $6,2 / 9,4$ & $6,6 / 9,6$ \\
\hline Mesosoma, largo & 10,4 & 12,3 & 15,4 & 14,7 \\
\hline Metasoma, largo & 25,8 & 27,9 & 45,8 & 40,6 \\
\hline Seg. I, largo / ancho & $3,0 / 3,6$ & $3,4 / 4$ & $5,3 / 5,6$ & $4,6 / 5,6$ \\
\hline Seg. II, largo / ancho & $3,5 / 3,1$ & $3,6 / 3,6$ & $6,3 / 5,0$ & $5,4 / 5,0$ \\
\hline Seg. III, largo / ancho & $3,6 / 3,0$ & $4 / 3,6$ & $6,6 / 4,8$ & $5,9 / 4,9$ \\
\hline Seg. IV, largo / ancho & $4,5 / 3,0$ & $4,5 / 3,4$ & $7,5 / 4,8$ & $6,5 / 4,8$ \\
\hline Seg. V, largo / ancho / alto & $5,0 / 3,7 / 2,2$ & $5,3 / 3,5 / 2,5$ & $9,0 / 4,8 / 3,5$ & $7,7 / 4,9 / 3,6$ \\
\hline Telson, largo & 6,2 & 7,1 & 11,1 & 10,5 \\
\hline Vesícula, largo / ancho / alto & $3,7 / 2,2 / 1,8$ & $4 / 2,5 / 2,1$ & $5,8 / 3,6 / 2,8$ & $5,5 / 3,4 / 2,8$ \\
\hline Aguijón, largo & 2,5 & 3,1 & 5,3 & 5,0 \\
\hline Pedipalpo, largo total & 15,7 & 15,7 & 27,4 & 22,9 \\
\hline Fémur, largo / ancho & $3,9 / 1,3$ & $3,7 / 1,5$ & $7,1 / 2,5$ & $5,7 / 2,2$ \\
\hline Patela, largo / ancho & $3,9 / 1,6$ & $3,9 / 1,8$ & $7,1 / 3,0$ & $6,1 / 2,7$ \\
\hline Pinza, largo/ ancho / alto & $7,9 / 2,3 / 2,7$ & $8,1 / 2,1 / 2,6$ & $13,2 / 4,0 / 4,5$ & $11,1 / 2,8 / 3,5$ \\
\hline Dedo móvil, largo & 4,1 & 4,3 & 7,1 & 6,6 \\
\hline
\end{tabular}



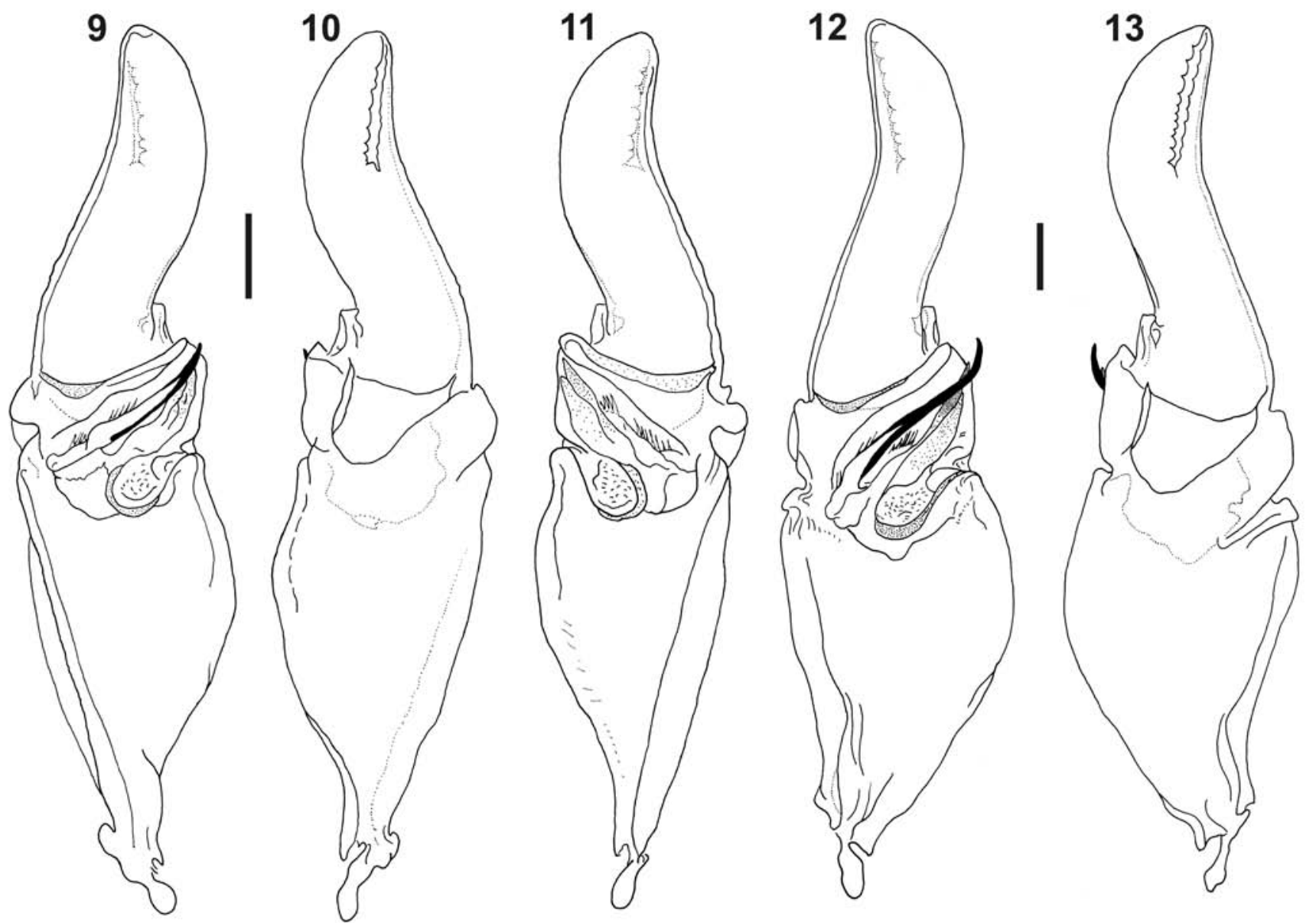

Figuras. 9-13. Hemispermatóforo. 9-11, Brachistosternus andinus Chamberlin, 1916, macho (MHNC). 9-10, hemiespermatóforo izquierdo; 9 , vista interna, 10, vista externa; 11, hemiespermatóforo derecho, vista interna. 12-13, Brachistosternus peruvianus Piza, 1974, macho (MHNC): hemiespermatóforo izquierdo; 12, vista interna, 13, vista externa. Escalas: $1 \mathrm{~mm}$.

patella sin carenas evidentes, superficies lisas. Pinza más robusta en los machos, con apófisis espiniforme en la cara interna, carena VM ligeramente evidente (sin gránulos), dedo móvil con 7-8 gránulos internos y 6-8 externos. Índice largo / ancho de la pinza: $\widehat{o}, 3,00-3,53$ (media $=3,23 ; n=13)$; + , 3,52-3,88 (media $=3,76 ; n=10)$. Índice largo / alto de la pinza: $\widehat{\jmath}, 2,58-2,92$ $($ media $=2,74 ; n=13) ;$ , 3,00-3,20 (media $=3,12 ; n=10)$.

Tricobotriotaxia: Patrón típico del subgénero Ministernus, tipo $\mathrm{C}$ con neobotriotaxia aditiva: fémur con 3 tricobotrias, una externa $(e)$, una dorsal $(d)$, una interna $(i)$. Patela con 18 tricobotrias, 3 ventrales $\left(v_{1}-v_{3}\right) ; 12$ externas, falta la tricobotria $e s b_{2}\left(e t_{1}-e t_{3}, e s t, e m_{1}, e m_{2}, e s b_{1}, e b_{1}-e b_{5}\right)$; dos dorsales $\left(d_{1}, d_{2}\right)$; una interna $(i)$. Pinza con 33 a 35 tricobotrias: 9-11 ventrales $\left(V_{1}-V_{11}\right) ; 12$ externas en la mano, con $5 E b\left(E t_{1}-E t_{5}, E s t, E s b\right.$, $\left.E b_{1}-E b_{5}\right)$; tres dorsales en la mano $(D b, D t, d b)$; cuatro externas en el dedo fijo (et, est, esb, eb); tres dorsales y dos internas en el dedo fijo $(d t, d s t, d s b, i t, i b)$. Número de tricobotrias ventrales de la pinza del pedipalpo ( $n=26)$ : $9(n=3), 10(6), 11(17)$.

Patas: Tegumento liso, sin presencia de carenas. Número de setas en el Tarso III $(n=33)$ : dorsales del telotarso: $5(n=1), 6$ (15), 7 (17); lateroventrales del telotarso: 4 (33); dorsales del basitarso: 4 (4), 5 (29). La fórmula más frecuente fue 7-4-5 en $16 / 33$ casos.

Tergitos: Tergitos I-VI finamente granulosos en $\widehat{\partial}$, en $q$ es liso. Tergito VII granuloso, evidenciando 4 carenas.
Peines: Número de dientes pectíneos: 27-30 (ð̋), 24-28 (ㅇ). Frecuencia: $\widehat{\jmath}(n=34): 27(n=7), 28(9), 29(11), 30(7)$; 우 $(n=$ 24), 24 (1), 25 (8), 26 (11), 27 (2), 28 (2).

Esternitos: lisos en las hembras (en los machos, al igual que otras especies de Brachistosternus con granulación roma en toda la extensión del segmento).

Metasoma: Segmentos caudales I-IV: carenas ausentes u obsoletas con gránulos pequeños, ligeramente menos evidentes en las hembras; carena Di completa en I-II, presente en la mitad distal en el segmento III y con algunos gránulos distales en el segmento IV. Carenas LM completa en el segmento I, presente en el tercio distal del segmento II y con unos pocos gránulos en III-IV. Carenas Lim sólo están en la mitad distal del segmento, con algunos gránulos en II-III y se encuentran ausentes en IV. Carenas Vl y Vsm ausentes. Existe granulación abundante (más evidente en el segmento I) en el espacio entre las carenas DL-LM, y algunos gránulos dispersos entre las LM-Lim. Cara ventral de los segmentos I-III con una fina granulación roma en $\widehat{\jmath}$. Segmento caudal V (figs. 2-4): cara dorsal con algunos gránulos en la posición de las carenas DL; caras laterales con gránulos esparcidos (en $q$ hay pocos gránulos), con una línea de setas en posición de la carena LM. Carenas VL presentes en los tres cuartos distales y con gránulos mayores en el tercio distal. Faz ventral granulosa en los dos tercios distales (puede apreciarse en algunos ejemplares la carena VM confundida en medio de la 

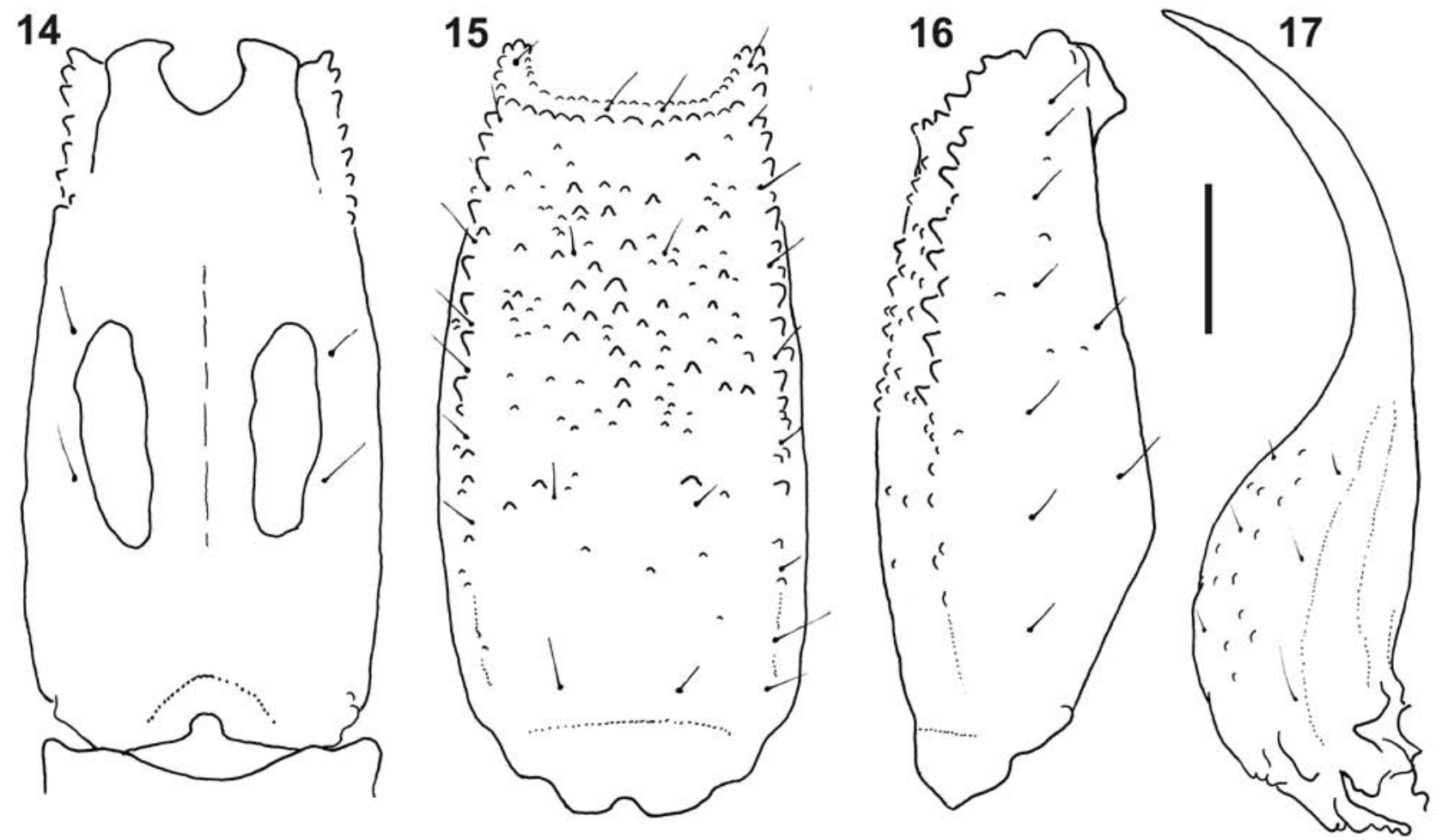

Figuras 14-17. Brachistosternus peruvianus Piza, 1974, macho (MHNC). 14-17. Segmento caudal V, 14, vista dorsal; 15, vista ventral, 16, vista lateral. 17. Telson, vista lateral. Escala $2 \mathrm{~mm}$.

granulación); carenas Vsm ausentes. Glándulas caudales pequeñas ubicadas en la parte central del segmento (fig. 2). Número de setas del segmento V: dorsales laterales $(n=46): 1 \quad(n=23)$, 2 (23); laterales $(n=46): 7(7), 8(18), 9(13), 10(8)$; ventrales laterales $(n=46): 7(3), 8(7), 9(36)$; ventrales $(n=23): 5(2)$, 6 (21), siendo la fórmula más frecuente $2-2-2$ en $21 / 23$ casos.

Telson: con algunos gránulos ventrales, aguijón corto (fig. 5). Alto relativo del telson: índice largo / alto: $\delta$, 3,43-3,95 (media= $3,68 ; n=12)$; + , 3,13-3,41 (media $=3,25, n=10)$

Hemiespermatóforo (figs. 9-11): lámina distal ligeramente corta, lóbulo distal corto. Apófisis cilíndrica sobrepasa ligeramente el lóbulo interno, espinas en hilera bien desarrolladas, triángulo basal en forma de una prolongación digitiforme cubierta de espinas cortas.

Distribución y hábitat. Se distribuye en valles y quebradas interandinas del departamento del Cusco entre los 2780 a 3350 m (fig. 1). Todas las localidades corresponden a la cuenca del río Vilcanota principalmente en el Valle Sagrado de los Incas. Biogeográficamente el área de distribución corresponde al "Distrito de Queshwa” de Ceballos Bendezú (1976) o la "Provincia Serrana” de Marín Moreno (1961), caracterizado por tener un clima templado, con seis meses de lluvias, la vegetación es arbustiva y subarbustiva. Los ejemplares capturados fueron encontrados principalmente debajo de piedras, generalmente medianas, en lugares donde existe poca vegetación; son abundantes en lugares donde hay piedras amontonadas y en los cercos de piedra cerca de los terrenos de cultivo. En la zona de Huacarpay se pudo apreciar a ejemplares de esta especie consumiendo dipluros. Por otro lado en algunas telas de la arańa viuda negra (Latrodectus sp.) se pueden encontrar restos de este escorpión. Brachistosternus andinus puede ser encontrado junto con Tityus footei Chamberlin, 1916 (Buthidae), Orobothriurus wawita Acosta \& Ochoa, 2000 (Bothriuridae) y Hadruroides mauryi Francke \& Soleglad, 1980 (Iuridae). Debido a que Maury (1978) confundió B. andinus y $B$. peruvianus, a las cuales trató como una sola entidad, la distribución que ofrece en dicho trabajo corresponde a la suma de ambas especies.

Material estudiado. PERÚ: Departamento Cusco: Provincia Urubamba: Ollantaytambo, [1315'15"S 72¹5'45”W, $2800 \mathrm{~m}$ ], 15 noviembre 1979, O. Ochoa M., 3 ㅇ (MHNC); igual localidad, 1-4 marzo 1947, J.C. Pallister, 1 juv. (AMNH); Wayracpunco, Ollantaytambo, [13¹4'46"S 72¹7'32”W, 2780 m], 20 marzo 1994, J.C. Chaparro \& J.A. Ochoa, 2 స, 3 ㅇ (MHNC); Maras, 1319'49"S 7209'23”W, 3350 m, 01 noviembre 1992, O. Mujica, 7 đ̊, 4 ㅇ (MHNC); igual localidad,

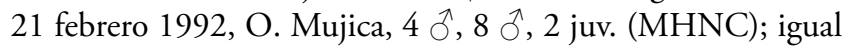
localidad, 26 febrero 1994, O Mujica, 3 ○ (MHNC); igual localidad, 09 setiembre 1992, J.A. Ochoa, 1 juv. (MHNC); igual localidad, 29-30 octubre 1997, O. Mujica \& J.A. Ochoa, 3 으 (MHNC); Pumahuanca, 1317'19”S 7207'35”W, 3000 m, 29 febrero 1992, J.A. Ochoa, 01 + (MHNC); Cattan, camino a Pumahuanca, 1317'52"S 7207'01'W, 2910 m, 02 enero 2000, J.A. Ochoa, 1 ๙ै (MHNC). Provincia Calca: Hacienda Urco, ca. Calca, [1319'35”S 71058'57”W, 2945 m], 15 setiembre 1939, K.P. \& J. M. Schmidt, 1 ô, 1 क (FMNH); Cerca a Písac, 13²5'36"S 7151'22”W, 3116 m, 09 enero 1999, J.A. Ochoa, 1 + (MHNC); Písac, 13²5’04”S 71050’58”W, 3000 m, 04 noviembre 1997, J.A. Ochoa, 1 ๙ (MHNC). Provincia Quispicanchi: Huacarpay, ca. Lucre, [13³6’30"S 7144’01”W, 

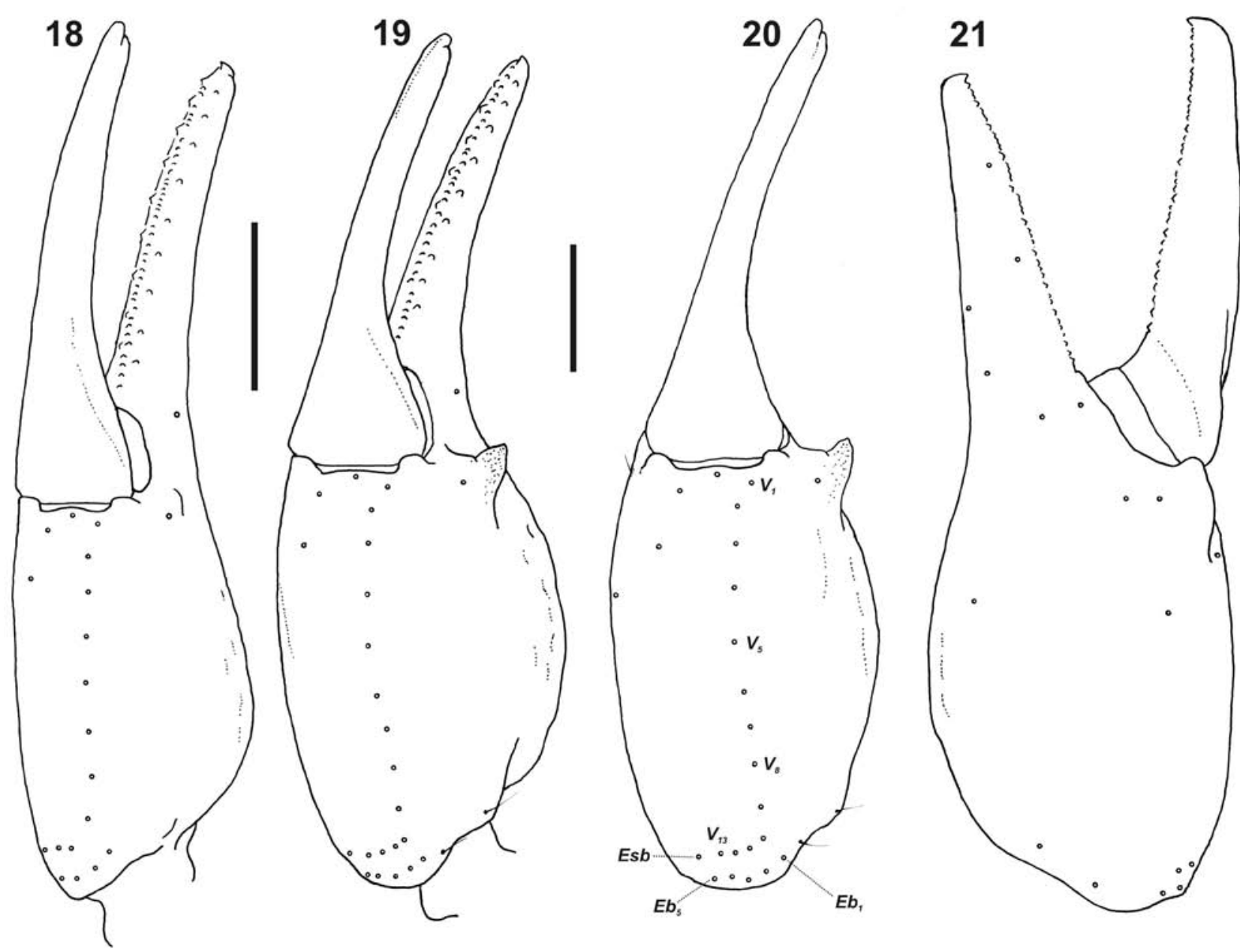

Figuras 18-21. Brachistosternus peruvianus Piza, 1974. 18, hembra (MHNC), pinza del pedipalpo derecho, vista ventral interna. 19-21, macho (MHNC), pinza del pedipalpo derecho. 19, vista ventromedial; 20, vista ventral, 21, vista externa. Escala $1 \mathrm{~mm}$.

3100 m], 24 abril 1994, O. Ochoa M. \& J.A. Ochoa, 10 ejm. (MHNC); igual localidad, 21 mayo 1994, O. Ochoa M., 1 §ૈ, 6 O (MHNC); igual localidad, 23 noviembre 1996, G. Vilcahuaman \& E. Minaya, 1 กิ, 1 ㅇ (MHNC); igual localidad, 27 setiembre 1992, O. Mujica, 1 (MHNC); igual localidad, 23 noviembre 1996, B. Quispe \& A. Povea, 1 I , 3 ㅇ (MHNC); igual localidad, 09 octubre 1993, O. Ochoa M. \& J.A. Ochoa, 5 ก, 14 ㅇ, 5 juv. (MHNC); iguales datos, 2 ô, 2 (CDA 164); Piquillacta, [1336'59"S 71\%42'56”W, $3170 \mathrm{~m}$ ] 08 octubre 1982, O. Ochoa M., 13 ejm. (MHNC); Lucre, [13³8’04”S 71044'10"W, 3100 m, 24 enero 1981, M. Del Castillo, 1 q (MHNC); Tipón, Oropesa, [13³4’22”S 7148’00”W, 3200 m aprox.], 05 noviembre 1992, J.A. Ochoa, 1 (MHNC); igual localidad, 14 enero 1999, 1 ๙ै, J.A. Ochoa (MHNC).

\section{Brachistosternus (Ministernus) peruvianus Piza, 1974}

(Figs. 12-21)

Brachistosternus peruvianus Piza, 1974: 155; Maury, 1978: 23. Brachistosternus (Microsternus) peruvianus: Francke, 1977: 75; Del Castillo, 1986: 91.

Brachistosternus (Microsternus) andinus: Maury, 1978 (part): 23-26 (error de determinación).

Brachistosternus (Ministernus) andinus: Lowe \& Fet, 2000: 52 (part) [erróneamente atribuyen la sinonimia de $B$. peruvianus con $B$. andinus a Maury (1978)].
Brachistosternus (Ministernus) peruvianus: Ochoa, 2003: 56; 2005: 54, 58, 62, fig. 4; Ojanguren Affilastro et al., 2007: 5, tabla 1; Ojanguren Affilastro \& Ramírez, 2009: 192, 195.

Brachistosternus peruvianus: Kovařík, 1998: 101; Ochoa \& Acosta, 2002: 2; Ochoa, 2005: 61, tabla 2; Rein, 2007: 3, 7; Ojanguren Affilastro \& Ramírez, 2009: 188, 192, figs. 1, 2, 3B, F.

Serie típica. 1 macho, 1 hembra sintipos (originalmente ESALQ). PERÚ: Departamento Apurímac [Cunyac o Pachachaca, nov. 1969], O. Ochoa M.

Diagnosis. Especie perteneciente al subgénero Brachistosternus (Ministernus); se diferencia de la especie más relacionada $(B$. andinus) por la mayor longitud de la apófisis cilíndrica y la forma más alargada de la lámina distal en el hemiespermatóforo (fig. 12). El número de dientes pectíneos y de tricobotrias ventrales en la pinza también es mayor en $B$. peruvianus (ver diagnosis correspondiente a $B$. andinus). Ambas especies ( $B$. andinus y $B$. peruvianus) se diferencian fácilmente de los otros dos integrantes del subgénero ( $B$. ferrugineus y $B$. simoneae) por la forma de la pinza del pedipalpo, que es mucho más delgada en las dos últimas especies. Adicionalmente $B$. ferrugineus presenta granulación fina en la cara ventral del segmento caudal $\mathrm{V}$ mientras que $B$. andinus y $B$. peruvianus presentan gránulos mayores ubicados en los dos tercios distales. En cuanto al hemiespermatóforo, $B$. ferrugineus presenta el triángulo basal con crestas esclerificadas, por el contrario en $B$. andinus y $B$. peruvianus el triángulo basal tiene forma digitiforme y cubierta de numerosas espinas cortas. 


\section{Redescripción}

Coloración y patrón de pigmentación: Color general desde amarillo-pajizo hasta amarillo-castaño, parte ventral ligeramente más clara, peines blanquecinos. El caparax puede estar despigmentado (salvo la cúpula ocular que es negruzca), o puede presentar manchas de pigmento en la parte central (alrededor de la cúpula ocular y hacia los laterales, donde pueden existir también algunas líneas de retículo). Tergitos I-VI en algunos casos sin pigmento, en otros con un pigmento tenue de forma irregular o con pigmento en los dos tercios anteriores. Pretergitos casi siempre pigmentados en su borde posterior. Tergito VII, esternitos, segmentos caudales, telson, patas y pedipalpos sin pigmento (excepto en algunos ejemplares que presentan un pigmento tenue en la cara ventral del segmento caudal V). Quelíceros con manchas cerca de la base de los dedos; dedos pigmentados.

Morfología: Longitud total: $\widehat{\partial}, 61,2-70,0 \mathrm{~mm}$; + , hasta 78,0 $\mathrm{mm}$. Medidas de un $\widehat{\partial}$ y una $\bigcirc$ (MHNC) en Tabla 1.

Queliceros: Con un diente subdistal en el dedo móvil. Tegumento liso con algunas setas ventrales en la base del dedo fijo.

Carapax: Borde anterior con una ligera prominencia mediana; surco longitudinal anterior completo pero no muy marcado, surcos longitudinal posterior y laterales posteriores más evidentes. Cúpula ocular desarrollada, ubicada en la parte central. Tegumento del caparax finamente granuloso en la parte central y hacia los laterales de la cúpula ocular, ángulos latero-posteriores con gránulos más notorios y abundantes, tercio anterior liso. En las hembras las granulaciones son menores.

Pedipalpos: (figs. 18-21): fémur con las carenas DI, DE, IM, VI obsoletas y con granulación menor; patela sin carenas evidentes, superficies lisas; pinza más robusta en los machos (figs. 18-19), carena VM marcada por un levantamiento del tegumento, pero sin granulación, $\widehat{\partial}$ con apófisis espiniforme en la cara interna; dedo móvil con un fila de gránulos en el borde medial de los dedos acompañada de 9 gránulos externos y 9-10 internos. Índice largo / ancho de la pinza: 0 ô, 3,26-3,40 (media= 3,32, $n=3)$; + , 3,83-4,17 (media=3.93, $n=5)$. Índice largo / alto de la pinza: $\hat{\jmath}, 2,82-2,97$ (media $=2,90 ; n=3)$; hembras, $3,15-3,57$ (media $=3,27 ; n=5)$.

Tricobotriotaxia: Patrón típico del subgénero Ministernus; Tipo $\mathrm{C}$ con neobotriotaxia aditiva: Fémur con 3 tricobotrias, una externa $(e)$, una dorsal $(d)$, una interna $(i)$. Patela con 18 tricobotrias, 3 ventrales $\left(v_{1}-v_{3}\right) ; 12$ externas, falta la tricobotria $e s b_{2}\left(e t_{1}-e t_{3}, e s t, e m_{1}, e m_{2}, e s b_{1}, e b_{1}-e b_{5}\right)$; dos dorsales $\left(d_{1}, d_{2}\right)$; una interna $(i)$. Pinza con 35 a 37 tricobotrias: 11-13 ventrales $\left(V_{1}-V_{13}\right) ; 12$ externas en la mano, con $5 E b\left(E t_{1}-E t_{5}, E s t, E s b\right.$, $\left.E b_{1}-E b_{5}\right)$; tres dorsales en la mano $(D b, D t, d b)$; cuatro externas en el dedo fijo (et, est, esb, eb); tres dorsales y dos internas en el dedo fijo $(d t, d s t, d s b, i t, i b)$. Número de tricobotrias ventrales de la pinza del pedipalpo $(n=12): 11(n=4), 12(4), 13(4)$.

Patas: tegumento liso, sin presencia de carenas. Número de setas en el Tarso III $(n=20)$ : dorsales del telotarso: $6(n=2), 7(18)$; lateroventrales del telotarso: 4 (17), 5(3); dorsales del basitarso: 4 (1), 5 (19). La fórmula más frecuente fue 7-4-5 en 14/20 casos.

Tergitos: I-VI con granulación evidente cerca al borde posterior y hacia los laterales. Tergito VII bien granuloso, donde se evidencian 4 carenas.
Peines: Número de dientes pectíneos: 35-39 (つ), 34-36 (Q); el número de dientes mencionado por Piza (1974) en la descripción original es 35-40. Frecuencia: $\widehat{\partial}(n=10): 35(n=1)$, $36(1), 37(4), 38(3), 39(1)$; 우 $(n=12), 34(4), 35$ (7), 36 (1).

Esternitos: Esternitos lisos en las hembras y en los machos con granulación roma que cubre toda su extensión.

Metasoma: Segmentos caudales I-IV: al igual que en B. andinus, la mayoría de las carenas están ausentes u obsoletas con granulación pequeña. Carenas DL completas en I-II, presentes en la mitad distal del segmento III, y sólo hay unos pocos gránulos en IV. Carenas Lm completas en I, presentes en la mitad distal en el segmento II, en el tercio distal en III y con 3 ó 4 gránulos distales en IV (en las hembras sólo hay 2 ó 3 gránulos distales en III y en IV está ausente). Carenas Lim presentes en la mitad distal en el segmento I, en el tercio distal en II, ausente o con pocos gránulos en III y ausente en IV (en las hembras está ausente en III-IV). Carenas VL y VSM ausentes. Espacio entre las carenas DL-LM y LM-Lim con granulación esparcida especialmente en los machos, la cual es más abundante en el segmento I y con pocos gránulos en IV (a veces sólo llega al segmento III). Segmento caudal V (figs. 14-16): cara dorsal sin granulación. Caras laterales en los machos con pocos gránulos esparcidos (en las hembras liso), carena LM evidenciada por la línea de setas laterales. Carena $V_{\mathrm{L}}$ presente en los tres cuartos distales, con los gránulos más evidentes en la parte distal. Cara ventral con granulación esparcida en los dos tercios distales; la carena VM no es evidente. Glándulas caudales elípticas ubicadas en la mitad del segmento (fig. 14). Número de setas del segmento caudal V, dorsales laterales $(n=20): 1(n=16), 2(4)$; laterales $(n=20)$ : $6(1), 7$ (18), $8(1)$; ventrales laterales $(n=20): 7(11)$, $8(8), 9(1)$; ventrales $(n=10): 5(1), 6(8), 7(1)$; fórmula ventral más frecuente $2-2-2$ en $8 / 10$ casos.

Telson: Superficie de la vesícula con gránulos en la cara ventral (fig. 17). Alto relativo del telson: índice largo / alto: §̂, 3,75-4,16 $($ media $=3.94, n=3)$; $9,3,52-3,84($ media $=3,65, n=4)$.

Hemiespermatóforo (figs. 12-13): lámina distal ligeramente alargada, un poco curvada en su parte media; la apófisis cilíndrica sobrepasa ampliamente la altura del lóbulo interno y de la apófisis laminar; concavidad capsular ligeramente extendida; conjunto de espinas de lóbulo basal, espinas en hilera y triángulo basal como en $B$. andinus.

Distribución y hábitat. Valles interandinos y cañones profundos en la cuenca de los ríos Apurímac y Pachachaca, en los departamentos de Cusco y Apurímac, desde los 1900 hasta los 2700 m (fig. 1). La zona comprende ambientes xerofíticos de clima cálido seco, lo que correspondería a los "valles interandinos cálidos” según Ceballos Bendezú (1976) o a la provincia de los "Montes Pluvifolios" (Marín Moreno 1961). Brachistosternus andinus es la especie cogenérica de distribución más cercana de $B$. peruvianus, pero ambas especies están separadas geográficamente por una "divisoria de aguas" (en su mayor parte por encima de los $4000 \mathrm{~m}$ de altitud). Esta divisoria corresponde a la cordillera de Vilcabamba, la cual divide dos de las más importantes cuencas del sur del Perú, la del río Apurímac (donde se encuentra B. peruvianus) y la cuenca del río Vilcanota donde se halla distribuido $B$. andinus. Las localidades mencionadas para $B$. andinus en el trabajo de Maury (1978) del departamento de Ayacucho deben ser sujetas a confirmación. En algunas localidades de Apurímac 
B. peruvianus fue hallado junto con Tityus footei y Pachakutej iskay (Acosta \& Ochoa, 2001).

Material estudiado.- PERÚ: departamento Apurímac: Provincia Abancay: Pachachaca, cerca al puente de piedra; aprox. 1900 m, 24 noviembre 1969, O. Ochoa M., 1 ภ, 4 ㅇ, 4 juv. (MHNC 112-114, 116-118, 123-125); igual localidad, 13039'15"S 72056'36”W, 2000 m, 14 noviembre 1997, O. Ochoa M. \& J.A. Ochoa, 2 ㅇ (MHNC); igual localidad, 23 diciembre 2007, J. Vitorino \& J.A. Ochoa, 1 đ (AMNH); Tamburco, 13036'56"S 72'52'33”'W, 2670 m, 15 noviembre 1997, O. Ochoa M. \& J.A. Ochoa, 2 ठ, 3 क (MHNC); Curawasi, 15 diciembre 1993, L. Valenzuela, 1 के (MHNC); Cconoc, [1331'49”S 72³8'55”W, 1900 m], 18 octubre 1992, O. Mujica \& J.A. Ochoa, 1 đ, 2 + 1 juv. (MHNC); iguales datos, $1 \hat{\jmath}$ (CDA 165). Departamento Cusco: Provincia Anta: ca. Limatambo [1331'49"S 72038'55"W, 2520 m], 05 abril 1992, J. Achicahuala, 1 ㅇ (MHNC).

\section{Agradecimientos}

Agradezco a Oscar Ochoa M. por proporcionarme información sobre la colección del MHNC; igualmente a Joyce Vitorino, John Achicahuala, Oscar Mujica y Juan C. Chaparro por su ayuda en los viajes de colecta. A la Fundação de Amparo à Pesquisa do Estado de São Paulo, Brasil por el soporte financiero (FAPESP 2010/00018-9). A Ricardo Pinto da Rocha por las facilidades en el Laboratorio de Aracnología de la Universidade de Sáo Paulo (IB/USP). Finalmente agradezco a la Dirección General de Forestal y Fauna de Perú (ex Instituto Nacional de Recursos Naturales de Perú (INRENA) por los permisos de colecta 061-2004-INRENA-IFFS-DCB, 106-2008-INRENAIFFSDCB, RD-0157-2010-AG-DGFFS-DGEFFS.

\section{Literatura citada}

Acosta L.E. \& E.A. Maury. 1998. Scorpiones. En: J.J. Morrone \& S. Coscaron, eds. Biodiversidad de Arthropodos argentinos. Una perspectiva biotaxonómica. Ediciones Sur, La Plata. Pp. 545-559

Acosta L.E. \& J.A. Ochoa. 2001. Two new species of Orobothriurus Maury, 1976 from Argentina and Peru, with comments on the systematics of the genus (Scorpiones: Bothriuridae). In: V. Fet \& P.A. Selden, eds. Scorpions 2001. In memoriam Gary A. Polis. Burnham Beeches, Bucks: British Arachnological Society. Pp. 203-214.

Acosta L.E. \& J.A. Ochoa. 2002. Lista de los escorpiones bolivianos (Chelicerata: Scorpiones), con notas sobre su distribución. Revista de la Sociedad Argentina de Entomología 61 (3-4): 15-23.

Aguilar P.G. \& O. Meneses. 1970. Escorpiones y Escorpionismo en el Perú I: Nota preliminar sobre los Scorpionida peruanos. Anales Científicos de la Universidad Nacional Agraria La Molina 8: 1-5.

Ceballos Bendezú I. 1976. Nuevo esquema biogeográfico del Perú. Revista Universitaria, Universidad Nacional del Cusco 130: 19-44.

Chamberlin R.V. 1916. Results of the Yale Peruvian Expedition of 1911. The Arachnida. Bulletin of the Museum of Comparative Zoology 60 (6): 177-299.

Del Castillo M. 1986. Contribución al Conocimiento de la escorpiofauna de los departamentos de Cusco y Apurímac. Revista de Zoología, Cusco 2: 89-92.

Francke O.F. 1977. Escorpiones y Escorpionismo en el Perú VI: Lista de especies y claves para identificar las familias y los géneros. Revista Peruana de Entomología 20: 73-76.
Kovařík F. 1998. Štiři (Scorpions). Madagaskar, Jihlava, 175 pp. [Czech].

Lourenço W.R. 2000. Confirmation de la présence du genre Brachistosternus Pocock (Scorpiones, Bothriuridae) au Brésil et description d'une espèce nouvelle. Revue Arachnologique 13(6): 93-100.

Lowe G. \& V. Fet. 2000. Family Bothriuridae Simon, 1880. In: V. Fet, W.D. Sissom, G. Lowe \& M.E. Braunwalder, eds. Catalog of the Scorpions of the World (1758-1998). The New York Entomological Society, New York. Pp. 17-53.

Marín Moreno F. 1961. Panorama fitogeográfico del Perú. Revista Universitaria, Universidad Nacional del Cusco 120: 9-68.

Maury E.A. 1973. Las tricobotrias y su importancia en la sistemática del género Brachistosternus Pocock, 1894 (Scorpiones, Bothriuridae). Physis, Sec. C 32(85): 247-254.

Maury E.A. 1974. Escorpiofauna chaqueña 1. La verdadera identidad de Brachistosternus (Microsternus) ferrugineus (Thorell 1876) (Bothriuridae). Physis, Sec. C 33(86): 73-84.

Maury E.A. 1978. Escorpiones y escorpionismo en el Perú VII. Nuevos hallazgos y redescripción de Brachistosternus (Microsternus) andinus Chamberlin, 1916 (Bothriuridae). Revista Peruana de Entomología 21(1): 23-26.

Maury E.A. 1979. Apuntes para una zoogeografía de la escorpiofauna Argentina. Acta Zoológica Lilloana 35(2): 703-719.

Mello-Leitão C. de. 1931. Notas sôbre os Bothriuridae Sul-Americanos. Arquivos do Museu Nacional 33: 75-105.

Mello-Leitão C. de. 1932. Notas sôbre escorpiões Sul-Americanos. Arquivos do Museu Nacional 34: 9-46.

Mello-Leitão C. de. 1945. Escorpiáes sul-americanos. Arquivos do Museu Nacional 40: 7-468.

Ochoa J.A. 2002 [2003]. Nueva especie de Brachistosternus Pocock (Scorpiones: Bothriuridae) del Sur del Perú. Revista Peruana de Biología 9(2): 55-63.

Ochoa J.A. 2005. Patrones de distribución de escorpiones de la región andina en el sur peruano. Revista Peruana de Biología 12(1): 49-68.

Ochoa J.A. \& L.E. Acosta. 2002. Two New Andean Species of Brachistosternus Pocock (Scorpiones: Bothriuridae). Euscorpius 2: 1-13.

Ochoa J.A., R. Botero-Trujillo \& L. Prendini. 2010. On the troglomorphic scorpion Troglotayosicus humiculum (Scorpiones, Troglotayosicidae), with first description of the adults. American Museum Novitates 3691: 1-19.

Ochoa J.A. \& L. Prendini. 2010. The genus Hadruroides Pocock, 1893 (Scorpiones: Iuridae), in Peru: New records and descriptions of six new species. American Museum Novitates 3687: 1-56.

Ojanguren Affilastro A.A., C.I. Mattoni \& L. Prendini. 2007. The genus Brachistosternus (Scorpiones: Bothriuridae) in Chile, with descriptions of two new species. American Museum Novitates 3564: 1-44.

Ojanguren Affilastro A.A. \& M.J. Ramírez. 2009. Phylogenetic analysis of the scorpion genus Brachistosternus (Arachnida, Scorpiones, Bothriuridae). Zoologica Scripta 38 (2): 183-198.

Piza S. de Toledo. 1974. A new scorpion from Peru (Bothriuridae). Revista de Agricultura, Piracicaba, São Paulo 49(4): 155-256.

Prendini L. 2000. Phylogeny and classification of the superfamily Scorpionoidea Latreille 1802 (Chelicerata, Scorpiones): an exemplar approach. Cladistics 16: 1-78.

Rein J.O. 2007. Taxonomic updates in scorpions (Arachnida: Scorpiones) since the publication of the Catalogue of the Scorpions of the World (1758-1998) (Fet, Sissom, Lowe, and Braunwalder, 2000). Part 1: Bothriuridae. The scorpion Files - Occasional Papers 1: 1-12. (http://www.ub.ntnu. no/scorpion-files/sf_occationalpapers.php) 
Sissom W.D., G.A. Polis \& D.D. Watt. 1990. Field and laboratory methods. In: Polis, G. A. (ed.), The Biology of Scorpions. Stanford University Press, Stanford, California. Pp. 445-461.

Stahnke H.L. 1970. Scorpion nomenclature and mensuration. Entomological News, 81: 297-316.
Vachon M. 1952. Étude sur les Scorpions. Alger: Institut Pasteur d'Algérie, $482 \mathrm{pp}$.

Vachon M. 1973 [1974]. Étude des caractères utilisés pour classer les familles et les genres de scorpions (Arachnides). 1. La trichobothriotaxie en arachnologie. Sigles trichobothriaux et types de trichobothriotaxie chez les scorpions. Bulletin du Muséum National d'Histoire Naturelle (Paris) Ser. 3, 140: 857-958. 